\title{
RECURSIVE CONSTRUCTIONS AND SOME PROPERTIES OF TWOFOLD DESIGNS WITH BLOCK SIZE FOUR
}

\author{
K. T. PHELPS and A. ROSA \\ (Received 16 May 1986; revised 10 September 1986) \\ Communicated by Louis Caccetta
}

\begin{abstract}
A direct construction for partially resolvable $t$-partitions is presented and then used to give a recursive construction for $\operatorname{BIBDs}(v, 4,2)$. In particular, we construct $\operatorname{BIBD}(v, 4,2)$ with $\operatorname{BIBD}(u, 4,2)$ embedded in it whenever $v=3 u+a, a \in\{1,4,7\}$. This result allows us to give simple proofs for the existence of $\operatorname{BIBD}(v, 4,2)$ with various additioinal properties.
\end{abstract}

1980 Mathematics subject classification (Amer. Math. Soc.): 05 B 05.

\section{Introduction}

A $t-(v, K, \lambda)$-partition (or briefly, a $t$-partition) is a pair $(V, B)$ where $V$ is a $v$-set and $B$ is a collection of $k_{i}$-subsets of $V$ (called blocks) with $k_{i} \in K$ such that every $t$-subset of $V$ is contained in exactly $\lambda$ blocks of $B$. (A $t$-partition is sometimes called a $t$-wise balanced design.) The elements of $K$ are the block sizes.

Given a $t-(v, K, \lambda)$-partition $(V, B)$, a $q$-fold parallel class (a $q$-PC) is a subset $P$ of $B$ such that each element of $V$ is contained in exactly $q$ blocks of $P$. A 1-fold parallel class is called simply a parallel class (PC); it is a subset of $B$ which partitions $V$.

The research of the first author was conducted while he was a visiting professor, University of Waterloo, Department of Combinatorics \& Optimization.

The research of the second author was supported by NSERC No. A7268.

(c) 1988 Australian Mathematical Society 0263-6115/88 \$A2.00+0.00 
Generalizing slightly from [5], we have the following definition:

A partially resolvable t-partition PRP $t-(v ; P, S ; \lambda ; M)$ is a $t-(v, K, \lambda)$ partition such that

(i) $K=P \cup S, P \cap S=\varnothing$,

(ii) $|P|=|M|$,

(iii) if $P=\left\{p_{1}, \ldots, p_{u}\right\}, M=\left\{m_{1}, \ldots, m_{u}\right\}$, the blocks of size $p_{i}$ can be partitioned into $m_{i} \lambda$-fold parallel classes.

Here $t \geqslant 2$, and $p, s \geqslant t$ for every $p \in P, s \in S$.

In this paper, we will be concerned with the special cases $t=2, P=\{3\}$, $S=\{4\}, \lambda=2$, and $M=\{m\}$ where $m=(v-4) / 2$ or $(v-7) / 2$. We denote the corresponding PRPs by PRP $2-(v ; 3,4 ; 2, m)$. In Section 2 , we determine completely the spectrum for PRP $2-(v ; 3,4 ; 2, m) \mathrm{s}$ with $m=(v-4) / 2$ or $m=(v-7) / 2$. In Section 3, we apply the obtained PRPs to obtain some recursive constructions for twofold block designs with block-size $k=4$ (that is, for $\operatorname{BIBD}(v, 4,2)$; t the notation $S_{2}(2,4, v)$ is also used for these designs but the latter notation is sometimes understood to indicate that no repeated blocks are allowed). Finally, in Section 4 we indicate applications of these results to the existence of twofold designs with $k=4$ having some additional properties

2. The existence of $P R P 2-(v ; 3,4 ; 2 ; m) \mathrm{s}, m \in\{(v-4) / 2,(v-7) / 2\}$

In this section, we denote PRP $2-(v ; 3,4 ; 2, m)$ briefly by $P(v, m)$. Here $m=m_{1}=(v-4) / .2$ or $m=m_{2}=(v-7) / 2$.

If a $P\left(v, m_{1}\right)$ exists then $v \equiv 0(\bmod 2)$; also $($ if $v>4), v \equiv 0(\bmod 3)$ so $v \equiv 0$ (mod6). It follows that the number of blocks of size 4 is $v / 2$. Similarly, if a $P\left(v, m_{2}\right)$ exists then $v \equiv 1(\bmod 2)$, and $($ if $v>7)$, also $v \equiv 0(\bmod 3)$, thus $v \equiv 3$ (mod 6), and it follows that the number of blocks of size 4 is $v$.

We will show that these necessary conditions for existence are also sufficient. When $m=m_{1}$, we distinguish two cases.

LEMMA 2.1. $A P\left(v, m_{1}\right)$ exists for all $v \equiv 0(\bmod 12)$.

Proof. Let $v \equiv 0(\bmod 12), v=12 s$. Let $V=Z_{4 \varepsilon} \times Z_{3}$, and let the base blocks for $B$ be as follows (the base blocks are to be developed modulo $(4 s, 3)$ ):

$$
\begin{aligned}
& \{(0,0),(1,0),(2 s, 0),(2 s+1,0)\}, \\
& \left.\begin{array}{l}
\{(0,0),(r, 1),(2 s-r, 1)\} \\
\{(0,0),(r, 2),(2 s-r, 2)\}
\end{array}\right\}, \quad r=1,2, \ldots, s-1 \text {, }
\end{aligned}
$$



(4)
(a) $\{(0,0),(2 s, 1),(2 s+1,1)\},\{(0,0),(-(s-1), 2),(s, 2)\}$,
(b) $\{(0,0),(s, 1),(2 s, 2)\},\{(0,0),(-(s-1), 1),(-(2 s-1), 2)\}$,
(c) $\{(0,0),(0,1),(0,2)\}$ taken twice.

Let us first verify that $(V, B)$ is a pairwise balanced design with $\lambda=2$. For this we have to show that each difference, pure or mixed, occurs precisely twice among the differences in the base blocks. The pure difference $1 \bmod 3$ occurs in (4) (c). As for pure differences mod $4 s$, differences 1 and $2 s-1$ occur once in (1) and once in (4)(a) while difference $2 s$ occurs twice in (1). Each of the even pure differences $2,4, \ldots, 2 s-2$ occurs twice in (2), and each of the pure odd differences $3,5, \ldots, 2 s-3$ occurs twice in (3). As for mixed differences, the blocks of (2) contain each of them, except for $0,+s,-s$ and $2 s$, exactly once. Similarly, the blocks of (3) contain each mixed difference, except for $0, \pm s, \pm(s-1)$, $\pm(2 s-1)$ and $2 s$ exactly once. The blocks of (4)(a) contain the mixed differences $-(2 s-1), 2 s$, and $s-1,-s$ while the blocks of (4)(b) contain the mixed differences $s, s, 2 s$ and $-(s-1),-s, 2 s-1$.

To verify that we have a PRP, note that the blocks of (1) yield the required $v / 2$ blocks of size 4 . The blocks of (2) and (3) yield 3(s-1) and 3(s-2) 2-PCs respectively. Finally, (4) yields 72 -PCs, as (a) and (b) yield three 2-PCs each, and (c) yields one 2-PC. Note, however, that by combining the orbits of (c) with two of the orbits of (b) we can ensure that no 2-PC will contain repeated triples.

LeMMA 2.2. $A P\left(v, m_{1}\right)$ exists for all $v \equiv 6(\bmod 12)$.

Proof. Let $v \equiv 6(\bmod 12), v=12 s+6, s \geqslant 0$. Let $V=Z_{6 \varepsilon+3} \times\{1,2\}$. Let $P_{1}^{i}, P_{2}^{i}, \ldots, P_{3 \varepsilon+1}^{i}$ be the parallel classes of a Kirkman triple system of order $6 s+3$ on $Z_{6 \varepsilon+3} \times\{i\}, i=1,2$ (such a system is known to exist for all integers $s \geqslant 0$ [7]). Without loss of generality, let the PC $P_{1}^{1}$ be $\left\{0_{1},(2 s+1)_{1},(4 s+2)_{1}\right\}$ mod $6 s+3$ (here and further we write for brevity $x_{i}$ for an element $(x, i)$ ). Let the base blocks of $B$ be as follows (the base blocks are to be developed modulo $6 s+3)$ :

$$
\begin{gathered}
\left\{0_{1},(2 s+1)_{1}, s_{2},(s+1)_{2}\right\}, \\
\left\{0_{2}, i_{1},(6 s+3-i)_{1}\right\}, \quad i=1,2, \ldots, 3 s+1, \\
\left\{0_{1}, 0_{2},(2 s+2)_{2}\right\}, \\
\left\{0_{1},(2 s+2-i)_{2},(2 s+2+i)_{2}\right\}, \quad i=1,2, \ldots, s, \\
\left\{0_{1},(s-i)_{2},(3 s+2+i)_{2}\right\}, \quad i=1,2, \ldots,\lfloor(s-1) / 2], \\
\left\{0_{1},(5 s+2-i)_{2},(5 s+3+i)_{2}\right\}, \quad i=1,2, \ldots,\lfloor 3 s / 2] .
\end{gathered}
$$


In addition to the blocks determined by base blocks above, let also $B$ contain:
(a) all triples of the parallel classes $P_{2}^{1}, P_{3}^{1}, \ldots, P_{3 s+1}^{1}$,

(b) all triples of the parallel classes $P_{1}^{2}, P_{2}^{2}, \ldots, P_{3 s+1}^{2}$.

Clearly, each of the pairs of elements occurs in exactly two triples of $B$. The 2-PCs are obtained by taking unions of a PC of (4)(a) with an orbit of (3) (there are $3 s$ such orbits), and unions of a PC of (4)(b) with an orbit of (2). This yields a total of $6 s+12$-PCs.

Again, no 2-PC contains repeated triples.

LeMMA 2.3. $A P\left(v, m_{2}\right)$ exists for all $v \equiv 3(\bmod 6)$.

Proof. Let $v \equiv 3(\bmod 6), v=6 s+3$, and assume $s \geqslant 6$. Let $V=Z_{2 \varepsilon+1} \times Z_{3}$, and let the base blocks of $B$ be as follows (the base blocks are to be developed $\bmod (2 s+1,3))$ :

$$
\begin{aligned}
& \{(0,0),(2,0),(8,0),(12,0)\}, \\
& \text { (a) }\{(0,0),(0,1),(0,2)\} \text { taken twice, } \\
& \text { (b) }\{(0,0),(1,1),(4,2)\},\{(0,0),(2,1),(6,2)\} \\
& \{(5,0),(3,1),(8,2)\},\{(6,0),(5,1),(0,2)\} \text {, } \\
& \{(0,1),(4 r-2,1),(2 r-1,0)\}\} \text { for } r=1,2,3 \text { once and } \\
& \{(0,2),(4 r, 2),(2 r, 0)\} \quad\} \text { for } r=4,5, \ldots, s / 2 \text { twice. }
\end{aligned}
$$

When $s$ is odd, take also

$$
\{(0,1),(2 s, 1),(s, 0)\},\{(0,2),(2 s, 2),(s, 0)\} .
$$

Let us verify that each difference, pure or mixed, occurs exactly twice among the differences in the base blocks. The pure difference 1 mod 3 occurs in triples of (2)(a). As for the pure differences mod $2 s+1$, the differences 2, 4, 6, 8, 10, 12 occur once in (1) and once in (3). The remaining pure differences occur in the triples of (3) (and (4) if $s$ is odd). The mixed differences $\pm 1, \pm 2, \pm 3, \pm 4, \pm 5$, \pm 6 occur once in triples of (2)(b), and once in triples of (3). The remaining mixed differences all occur in triples of (3) (and (4) if $s$ is odd).

The blocks of (2) give 7 2-PCs, while the blocks of (3) and (4) together give $3 s-9$ 2-PCs, for a total of $3 s-2$ 2-PCs. Clearly, by combining the orbits properly, we can avoid repeated triples in the 2-PCs.

Finally, we consider the cases whewre $1 \leqslant s<6$. For $s=1$, take a $\operatorname{BIBD}(10,4,2)$ and delete a point; $s=2$ take a $S_{1}(2,4,16)$, delete a point and add the blocks of a Kirkman triple system of order 15, that is, $\operatorname{KTS}(15) ; s=3$, take a 
$S_{1}(2,4,25)$, delete four points (one block) and add a $\operatorname{KTS}(21)$. For the case $s=4$, take a KTS(27) plus develop the following base blocks in $Z_{9} \times Z_{3}:\left\{0_{0}, 3_{0}, 1_{1}, 5_{1}\right\}$, $\left\{0_{0}, 3_{1}, 4_{1}\right\},\left\{0_{0}, 3_{2}, 1_{2}\right\}$ and $\left\{0_{0}, 0_{1}, 0_{2}\right\}$ (twice). For the last case $s=5$, take these as base blocks in $Z_{11} \times Z_{3}:\left\{0_{0}, 1_{0}, 4_{0}, 6_{0}\right\},\left\{0_{0}, 5_{1}, 6_{1}\right\},\left\{0_{0}, 4_{1}, 7_{1}\right\}\left\{0_{0}, 2_{2}, 9_{2}\right\}$, $\left\{0_{0}, 1_{2}, 10_{2}\right\},\left\{0_{0}, 3_{1}, 0_{2}\right\},\left\{0_{0}, 0_{1}, 0_{2}\right\}$.

\section{Recursions for twofold block designs with $k=4$}

Consider a $\operatorname{BIBD}(v, k, \lambda)$, say, $(V, B)$; it is said to be embedded in a $\operatorname{BIBD}(w, k, \lambda)$, say $(W, C)$ if $V \subseteq W$ and $B \subseteq C$.

Theorem 3.1. Let $(V, B)$ be a $\operatorname{BIBD}(v, 4,2)$. Then for $w=3 v+\alpha, \alpha \in\{1,4,7\}$, there exists a $\mathrm{BIBD}(w, 4,2)(W, C)$ such that $(V, B)$ is embedded in $(W, C)$.

Proof. This is well-known for $\alpha=1$ (see, for example [4]). Let $u=w-v$, and let $V=\left\{a_{1}, \ldots, a_{v}\right\}$; recall that $v \equiv 1(\bmod 3)$, as this is a necessary condition for the existence of $\operatorname{arBD}(v, 4,2)$. If $\alpha=4$ then $u=2 v+4 \equiv 0(\bmod 6)$, and by Lemmas $2.1,2.2$ there exists a PRP $2-(u ; 3,4 ; 2 ; v)$. If $\alpha=7$, then $u=2 v$ $+7 \equiv 3(\bmod 6)$, and by Lemma 2.3 there exists a PRP $2-(u ; 3,4 ; 2, v)$. In either case, let $P_{1}, P_{2}, \ldots, P_{v}$ be the 2-PCs of this PRP, and let $Q$ be the set of blocks of size 4. From the set of blocks $D=\left\{\left\{a_{i}, x, y, z\right\},\{x, y, z\} \in P_{i}\right.$, $i \in\{1, \ldots, v\}\}$, and put $C=B \cup D \cup Q$. Then $(W, C)$ is $\operatorname{BIBD}(w, 4,2)$ containing $(V, B)$.

One of the consequences of Theorem 3.1 is an easy proof by induction of the existence of $\operatorname{BIBD}(v, 4,2) \mathrm{s}$ for all $v \equiv 1(\bmod 3)$ (which, of course, has been proved by Hanani in the early sixties and is well-known; see, for example [4]). In the next section, we consider the existence of these designs with some additional properties.

\section{The existence of twofold block designs with $k=4$ having additional properties}

It is possible for the set of blocks of a $\operatorname{BIBD}(V, B)$ with $\lambda=2$ to contain two blocks $b, b^{\prime}$ which are identical as subsets of $V$; in this case, $b$ is said to be a repeated block. We can prove easily the following (see [9]).

THEOREM 4.1. $A \operatorname{BIBD}(v, 4,2)$ with no repeated blocks exists if and only if $v \equiv 1$ $(\bmod 3), v>4$. 
Proof. The necessity is obvious. Neither the unique $\operatorname{BIBD}(7,4,2)$ nor any of the three nonisomorphic $\operatorname{BIBD}(10,4,2)$ s has a repeated block. An example of a $\operatorname{BIBD}(13,4,2)$ and of a $\operatorname{BIBD}(16,4,2)$ without repeated blocks is given in [6] and [2], respectively. If $V=Z_{19}$, and $B=\{\{0,1,7,11\},\{0,2,3,14\},\{0,4,6,9\}$ mod 19$\}$ then $(V, B)$ is a $\operatorname{BIBD}(19,4,2)$ without repeated blocks. Let now $v \geqslant 22$, $v \equiv 1(\bmod 3)$ and assume a $\operatorname{BIBD}(u, 4,2)$ with no repeated blocks exists for all $u<v$. Put $u=(v-\alpha) / 3$ where $\alpha=1$ if $v \equiv 4(\bmod 9), \alpha=4$ if $v \equiv 7(\bmod 9)$, and $\alpha=7$ if $v \equiv 1(\bmod 9)$. In either case, $u \equiv 1(\bmod 3), u \geqslant 7$, and there exists a $\operatorname{BIBD}(u, 4,2)$ with no repeated blocks. By Lemma $2.1-2.3$, there exists a $\operatorname{BIBD}(v, 4,2)$ with no repeated blocks.

If $(V, B)$ is a $\operatorname{BIBD}(v, k, \lambda)$ such that $B=B_{1} \cup B_{2}$ where $\left(V, B_{1}\right),\left(V, B_{2}\right)$ is a $\operatorname{BIBD}\left(v, k, \lambda^{\prime}\right)$, and $\mathrm{a} \operatorname{BIBD}\left(v, k, \lambda^{\prime \prime}\right)$, respectively, with $1 \leqslant \lambda^{\prime}, \lambda^{\prime \prime}<\lambda$ then $(V, B)$ is said to be decomposable (sometimes also called reducible). Otherwise, it is indecomposable.

The existence of indecomposable $\operatorname{BIBD}(v, 4,2)$ s for all $v \equiv 1(\bmod 3), v \geqslant 7$, was proved in [1,2]; see also [8]. The constructions of Section 2 enable us to give a simple proof of this, even if we require, in addition, that the designs have no repeated blocks (see [9]).

TheOREM 4.2. An indecomposable $\operatorname{BIBD}(v, 4,2)$ with no repeated blocks exists if and only if $v \equiv 1(\bmod 3), v>4$.

Proof. A $\operatorname{BIBD}(v, 4,2)$ with $v=7,10$, or 19 is necessarily indecomposable. Example of an indecomposable $\operatorname{BIBD}(v, 4,2)$ with $v=13,16$ having no repeated blocks can be ofund in [6] and [2], respectively. The rest of the proof follows that of Theorem 4.1.

In a similar manner, one could prove the existence of $\operatorname{BIBD}(v, 4,2) \mathrm{s}$ with any property that is preserved by embedding, provided the existence of designs with this property can be proved for sufficiently many small orders by direct means.

On the other hand, the methods of this paper are quite incapable of settling the following problem which is - to the best of our knowledge-still open:

Does there exist for every order $v \equiv 1(\bmod 3), v \geqslant 7$, a $\operatorname{BIBD}(v, 4,2)$ with no nontrivial sub-BIBD $(u, 4,2)$ ? Designs with this property are sometimes called simple; by a trivial subsystem here is meant a subsystem of order 1 or $v$.

This last question may be asked also for $\operatorname{BIBD}(v, 4,1) \mathrm{s}$, that is, when $\lambda=1$. In fact, the reader may well ask whether constructions similar to those of Sections 2, 3 cannot be obtained for $\lambda=1$ ? As a matter of fact, recently A. E. Brouwer and L. D. Andersen [3] have shown that a PRP $2-(v ; 3,4 ; 1, m)$, where $v \equiv 3$ $(\bmod 6)$, and $m \in\{(v-13) / 2,(v-25) / 2\}$ exists provided $v$ is sufficiently large. But the spectrum for these PRPs remains undetermined. 


\section{References}

[1] E. J. Billington, 'Construction of some irreducible designs', Combinat. Math. IX, pp. 182-196 (Lecture Notes in Math. 952, Springer, 1981).

[2] E. J. Billington, 'Further constructions of irreducible designs', Congr. Numer. 35 (1982), 77-89.

[3] A. E. Brouwer and L. D. Andersen, personal communication.

[4] H. Hanani, 'Balanced incomplete block designs and related designs', Discrete Math. 11 (1975), 255-369.

[5] C. Huang, E. Mendelsohn and A. Rosa, 'On partially resolvable t-partitions', Ann. Discrete Math. 12 (1982), 169-183.

[6] R. Mathon and A. Rosa, 'Some results on the existence and enumeration of BIBDs', Dept. of Math. and Stat., McMaster University, Mathematics Report 125-Dec-85.

[7] D. K. Ray-Chaudhuri and R. M. Wilson, 'Solution of Kirkman's schoolgirl problem', Combinatorics, pp. 187-203 (Proc. Sympos. Pure Math., 19, Amer. Math. Soc. 1971).

[8] A. P. Street, 'A survey of irreducible balanced incomplete block designs', Ars Combin. 19A (1985), 43-60.

[9] C. C. Lindner and A. P. Street, 'Disjoint designs and irreducible designs without repeated blocks', Ars Combin. 21A (1986), 229-236.

School of Mathematics

Georgia Institute of Technology

Atlanta, Georgia 30332

U.S.A.
Department of Mathematics and Statistics

McMaster University

Hamilton, Ontario

Canada L8S 4K1 